35

\title{
Visual threats reduce blood-feeding and trigger escape responses in Aedes aegypti mosquitoes.
}

\author{
Nicole E. Wynne, Karthikeyan Chandrasegaran, Lauren Fryzlewicz \& Clément Vinauger*
}

Department of Biochemistry, Virginia Polytechnic Institute and State University, Blacksburg, VA 24061, USA.

Center for Emerging Zoonotic and Arthropod-borne Pathogens, Virginia Tech, Blacksburg, VA, 24061, USA.

Keywords: Disease vector insects, host defense, vision, neuroethology

*To whom all correspondence should be addressed:

Clément Vinauger

Department of Biochemistry,

Virginia Polytechnic Institute and State University

Blacksburg, VA 24061, USA

e-mail: vinauger@vt.edu 


\section{Summary Statement}

In isolation, visual stimuli programmed to mimic a human swat prevent mosquitoes from

blood-feeding by triggering take-offs and escape responses.

Abstract

The diurnal mosquitoes Aedes aegypti are vectors of several arboviruses, including dengue, yellow fever, and Zika viruses. To find a host to feed on, they rely on the sophisticated integration of olfactory, visual, thermal, and gustatory cues reluctantly emitted by the hosts. If detected by their target, this latter may display defensive behaviors that mosquitoes need to be able to detect and escape. In humans, a typical response is a swat of the hand, which generates both mechanical and visual perturbations aimed at a mosquito. While the neuro-sensory mechanisms underlying the approach to the host have been the focus of numerous studies, the cues used by mosquitoes to detect and identify a potential threat remain largely understudied. In particular, the role of vision in mediating mosquitoes' ability to escape defensive hosts has yet to be analyzed. Here, we used programmable visual displays to generate expanding objects sharing characteristics with the visual component of an approaching hand and quantified the behavioral response of female mosquitoes. Results show that Ae. aegypti is capable of using visual information to decide whether to feed on an artificial host mimic. Stimulations delivered in a LED flight arena further reveal that landed females Ae. aegypti display a stereotypical escape strategy by taking off at an angle that is a function of the distance and direction of stimulus introduction. Altogether, this study demonstrates mosquitoes can use isolated visual cues to detect and avoid a potential threat.

\section{Introduction}

Mosquitoes are responsible for transmitting disease-causing pathogens killing more than 700,000 people every year (World Health Organization, 2018). The underlying motivation for adult females of epidemiologically problematic mosquito species is their need for nutrients,

65 including proteins, contained in vertebrate blood to produce progeny. Therefore, the reproductive 66 fitness of female mosquitoes is not only directly linked to their ability to detect, locate and feed

67 on a host but also to survive interactions with a larger and potentially defensive organism. But 68 accessing a resource that is hidden under the skin of mobile and defensive hosts is not free of any 69 risks and, in addition to physiological stresses associated with the ingestion of blood, hosts display defensive behaviors aimed at deterring or killing mosquitoes (Wynne et al., 2020). 
Mosquitoes rely on the integration of multiple sensory inputs to find hosts, where olfactory cues gate and modulate responses to visual and thermal targets (Liu and Vosshall, 2019; San Alberto et al., 2021; van Breugel et al., 2015; Vinauger et al., 2019; Zhan et al., 2021). However, how mosquitoes detect defensive maneuvers from their hosts remains largely understudied. In humans, a typical response to biting insects is swatting. From the insect's perspective, a swat corresponds to a rapidly approaching appendage, most often a hand, on an interception course. When approaching, the appendage induces rapid air displacement (i.e., mechanical component) and a rapid expansion of an object in the mosquito visual field (i.e., visual component). Using a mammalian tail simulator, Matherne et al., showed that the airflow generated by the swinging of mammals' tails reduced the proportion of Aedes aegypti landing by 50\% (Matherne et al., 2018). In free flight, both Ae. aegypti and the nocturnal malaria vector Anopheles coluzzii, displayed rapid escape maneuvers when stimulated with an artificial swatter that induces airflow and visual cues (Cribellier et al., 2021), hinting at the role of visual cues in signaling threats. However, to our knowledge, no study has yet isolated visual cues from mechanical cues to characterize the role of vision in mosquito escape behaviors.

Utilizing visual cues to detect and assess defensive hosts would allow mosquitoes to categorize potential hosts as defensive from a safe distance or under highly turbulent conditions where air displacement might not be a reliable indicator of a threat. In addition, some species display a "pre-biting" rest (Reid, 1968) or "pre-attack" resting (Clements, 1999) behavior when the mosquito density increases around a target host (Tuno et al., 2003), which is known to increase the amount of host defensive behaviors (Day and Edman, 1984; Edman et al., 1972). With an increase in host defenses but ultimately an endless supply of blood, it would appear adaptive for the mosquito to wait until the host activity calms. During this "pre-biting" phase, among the cues available to mosquitoes to evaluate the host's defensive behaviors, vision would allow them to do so from a safe distance away and even under highly turbulent conditions where mechanical cues may be unreliable (Tuno et al., 2003; Tuno et al., 2017). In this context, we tested the hypothesis that day-time biting Ae. aegypti mosquitoes can use visual cues to detect potential threats and adjust their behavior accordingly. To test our hypothesis, we relied on a quantitative analysis of Ae. aegypti's behavior to investigate how they respond to predator-like looming (expanding) stimuli designed to mimic the visual properties of a slapping hand since humans are Ae. aegypti's preferred host (Christophers, 1960; Ponlawat and Harrington, 2005). 


\section{Materials and Methods}

\section{Mosquitoes}

Wild type Aedes aegypti mosquitoes (Rockefeller strain, MR-734, MR4, ATCC®, Manassas, VA, USA) were used throughout the experiments. The colony was maintained in a climatic chamber set at $25 \pm 1^{\circ} \mathrm{C}, 60 \pm 10 \%$ relative humidity $(\mathrm{RH})$, and under a 12-12h light-dark cycle. Cages of adults were fed weekly using an artificial feeder (D.E. Lillie Glassblowers, Atlanta, Georgia; $2.5 \mathrm{~cm}$ internal diameter) with heparinized bovine blood (Lampire Biological Laboratories, Pipersville, PA, USA) heated at $37^{\circ} \mathrm{C}$ using a circulating water-bath. Between blood-meals, mosquitoes were fed ad libitum with $10 \%$ sucrose. Eggs were collected from blood-fed females and hatched in deionized water. Larvae were reared in groups of 200 in covered pans $(26 \times 35 \times 4 \mathrm{~cm})$ containing deionized water and fed ad libitum with fish food (Hikari

114 Tropic 382 First Bites - Petco, San Diego, CA, USA). Pupae, in groups of 100, were isolated in 16 oz containers (Mosquito Breeder Jar, Bioquip Products, Rancho Dominguez, CA, USA) until emergence.

For all the experiments, 6-8 days old female mosquitoes were used. These females were kept in the presence of males, fed ad libitum with $10 \%$ sucrose until $24 \mathrm{~h}$ prior to the experiments, and were never blood-fed. This gave mosquitoes the time to mate in the containers before the experiments (random dissection of randomly selected females revealed that $95 \%$ of them had oocytes) and Ae. aegypti females of this age class are known to actively seek hosts for blood feeding (Grant and O'Connell, 2007; Tallon et al., 2019). All experiments were performed during the last four hours of the mosquitoes' subjective day, i.e., Zeitgeber Time (ZT) 8-12 (Eilerts et al., 2018; Taylor and Jones, 1969).

\section{Expanding stimuli}

To present ecologically relevant stimuli, we video-tracked a slapping hand on a collision course with a fictive mosquito located at the camera's position (GoPro Hero 6 in "linear" recording mode at $60 \mathrm{fps}$, GoPro, San Mateo, CA, USA). Tracking of the hand's width at the widest point below knuckle height over ten replicates provided an average duration and expansion velocity profile of a slap (ImageJ; National Institutes of Health, USA; Fig. 1). This information was used to program the symmetrical expansion of a dark square over a bright green 

squares have been shown in tethered Ae. aegypti females (Vinauger et al., 2019), and the response characteristics of classical models such as Drosophila melanogaster have been extensively analyzed (Tammero and Dickinson, 2002). Furthermore, in the programmable LED arena, a square allowed for the expansion of the stimulus along both dimensions with a higher step resolution than, for example, a disk. The expansion velocity of the fictive swats used throughout the experiment was kept constant at 28.9 horizontal degrees. ${ }^{-1}$ (LCD monitor) and 183.6 horizontal degrees.s ${ }^{-1}$ (LED arena) as seen from the center of the mosquito container. These virtual swats correspond to the expansion velocity of a hand at $80 \mathrm{~ms}$ and $40 \mathrm{~ms}$ before contact, respectively. The rationale behind this choice was to simulate earlier phases of the swat in the feeding assays and later moments (i.e., closer to impact) in the take-off experiments, as the distance from the display was greater in the feeding assays than in the take-off experiments (300 $\mathrm{mm}$ versus $118.5 \mathrm{~mm}$, respectively).

\section{Artificial blood-feeding assays}

\section{a) Procedure}

To quantify the effect of visual motion on the ability of Ae. aegypti females to land and feed on an artificial feeder, we performed blood-feeding assays in the presence of visual patterns generated with PsychoPy v3.0 displayed on a 29-inch $(73 \mathrm{~cm}$ diagonal) LED-backlit LCD monitor (UltraSharp 29 Ultrawide Monitor - U2917W, Dell, Round Rock, TX, USA). PsychoPy is a Python-based application that allows for the design of behavioral experiments with precise spatial and temporal control of stimuli (Peirce et al., 2019). Specifically, two patterns were generated: 1) a uniform green screen (negative control), and 2) expanding squares (treatment group) programmed to have the same duration as a human swat (Fig. 2A, Movie 1). Green, with a peak irradiance at $535 \mathrm{~nm}$, was chosen as a background color to allow comparisons with the conditions in the LED arena (peak irradiance: $571 \mathrm{~nm}$ ) (Fig. S1).

The mosquitoes used for these experiments $(21<\mathrm{n}<35)$ were isolated into clear glass cylinders $(10 \mathrm{x} 10 \mathrm{~cm})$ and starved from sucrose for 24 hours prior to the experiment. Mosquitoes were tested during the last 2 hours of the photophase (ZT 10-12). In the first hour of the experiment, the screen was kept uniformly green to allow mosquitoes to acclimate to the 
on the fabric mesh-lined top side of the glass cylinder. The feeder was warmed to $37 \square$ using a circulating water bath thirty minutes prior to the onset of the experiment. The warmed feeder was filled with $\sim 5 \mathrm{ml}$ of heparinized bovine whole blood (Lampire Biological Laboratories, Pipersville, PA, USA) fifteen minutes prior to the beginning of the experiment to allow the blood to heat up. The fabric mesh-lined side of the clear glass cylinder allowed mosquitoes to sense the heat and see the visual contrast of the feeder. The number of mosquitoes that landed and bloodfed on the feeder were recorded using a camera (Logitech C920, Logitech, Lausanne, Switzerland).

In the negative controls, the baseline levels of landing and feeding were quantified in front of a uniform green screen. In the first hour, mosquitoes were allowed to acclimatize to the experimental conditions including the uniform green background. In the second hour, after placing the blood feeder on a glass cylinder enclosure, the number of landings and feedings on the feeder were quantified. In the treatment groups, mosquitoes isolated in the glass cylinder enclosure were acclimatized to the uniform green screen in the first hour, following which the visual stimuli, i.e., expanding squares, were introduced every 0.8 seconds for a duration of 0.3 seconds. The positions of the looming squares displayed were randomly determined for each experiment trial to account for potential spatial bias. For this purpose, PsychoPy used an array of cartesian coordinates randomized using the rand function in Microsoft Excel.

The glass cylinders were kept in the camera's field of view throughout the two-hour duration of every experiment trial.

\section{b) Statistical analysis}

After every trial, the number of engorged females was quantified via visual inspection of their abdomen for the presence of blood, and the number of landings was quantified from the video recordings. These numbers were then compared between treatments as proportions (categorical fixed predictors with two levels: uniform background and looming squares). For the analysis, we used a Generalized Linear Model assuming a quasibinomial error distribution for the proportion of feeding and a Poisson error distribution for the number of landings per mosquito. The analysis was performed in $\mathrm{R}$ (version 3.6.2) using the packages lme4 (version 1.1- 
a) Procedure

Experiments were performed in an LED-based arena (sensu (Reiser and Dickinson, 2008)) consisting of an array of $96 \times 16$ LEDs subtending $360^{\circ}$ horizontally and $54^{\circ}$ vertically from the center of the arena. Individual mosquitoes were cold anesthetized on ice and separated in clear acrylic cylinders with a clear acrylic lid on the top and a fabric mesh lining at the bottom. These containers were kept in the climatic chamber for at least two hours to allow the mosquitoes to recover from the cold anesthesia. Containers were moved to the experimental room $\left(23 \pm 1^{\circ} \mathrm{C}, 45 \pm 5 \% \mathrm{RH}\right)$ thirty minutes before the start of the experiment to allow the mosquitoes to acclimatize to the ambient conditions. In every experimental trial that lasted for the expanding stimulus being introduced every minute at a randomized angle around the arena (Fig. 3A, Movie 2). The distance and the direction to the point of stimulus introduction were a function of both the mosquito's position in the arena at the time of introduction and the randomized angle of introduction. The behavior of mosquitoes in the experiment trials was recorded at 30fps with a camera (Logitech C920, Logitech, Lausanne, Switzerland).

\section{b) Statistical analysis}

Mosquitoes that did not move throughout the duration of the experiment $(\sim 7 \%$ of the tested mosquitoes) were determined "not active" and discarded from the analysis. Because of the

214 introduction of the stimulus were discarded from the analysis $(n=63)$. Based on these criteria, a 215 total of 551 stimuli introductions were conserved, and videos of active landed mosquitoes were 216 trimmed 30 seconds before and 30 seconds after each stimuli introduction. These one-minute 217 trimmed videos were then converted into image sequences and imported into Fiji ImageJ 218 (National Institutes of Health) for manual tracking, using the Manual Tracking plugin. The head 219 of each mosquito was tracked before, during, and after stimulus introduction. The stimuli edges 220 and center points were also tracked for each stimulus introduction. From these stimuli 221 coordinates, the mosquito-stimulus distance and the angle of stimulus introduction were 222 recorded. Distances were measured in pixels and the punctual conversions to mm provided 223 throughout the manuscript were obtained by reporting the measured diameter of the arena to its 224 measurement on the videos. 
In addition, to calculate the escape probability of mosquitoes we considered responsive mosquitoes as those that took off within 10 frames post stimulus introduction (i.e., $1 / 3$ of a second), but overall observed that all mosquitoes that responded to the stimulus took off within the duration of the stimulus presentation, and no take offs were observed post stimulus. Take offs past this time frame were not considered responses as mosquitoes would have been intercepted by the fictive object beyond that time.

A baseline probability of take-off was determined by repeating the experiments with the exception that no visual looming stimuli were introduced. All LEDs of the arena were kept ON for thirty minutes and the behavior of individualized mosquitoes $(n=10)$ was recorded with the same video camera as the treatment group. For each mosquito, ten time points were randomly selected within these 30 minutes, using the sample function in $\mathrm{R}$ (i.e., $\mathrm{N}=100$ ). At each of these times and for up to five seconds afterwards, spontaneous take-offs were quantified and used to calculate the baseline take-off probability in the absence of visual stimuli.

All data were saved as .csv and imported in R (version 3.6.2) and trajectories were rotated so that all stimuli introductions were fictively re-positioned at $180^{\circ}$. All the points before the stimulus introduction were considered pre-stimulus, while all the points after the stimulus introduction were considered post-stimulus. From this data, four response variables were

242 calculated by subtracting the pre-stimulus period from the post-stimulus period (to account for mosquitoes walking before stimulus introduction): linear and angular velocity, escape direction and displacement: $i$ ) the linear velocity was determined by the distance between consecutive 245 points divided by the sampling interval (pixels. $\sec ^{-1}$ ); $i$ ) The angular velocity was calculated as change in heading between consecutive points divided by the sampling interval (degrees. $\sec ^{-1}$ ); iii) The displacement (distance travelled) and $i v$ ) escape direction (degrees) were quantified by measuring differences between the mosquito's location at time of stimulus introduction and its location 20 frames post-stimulus. The effects of the distance and direction to the point of stimulus introduction on these four variables were assessed by means of Generalized Linear Models (GLMs) using the $R$ package lme4 (Bates et al., 2014).

In order to quantify the amount of digitizing error introduced by manual tracking of the mosquitoes, 3 experimenters tracked the same 2-second-long sequence (59 frames), once a day

254 during 3 consecutive days. This provided an estimate of the inter- and intra-individual errors in 
the digitized cartesian coordinates, quantified here as the Root Square Mean Error (RMSE), calculated according to equation (1):

$$
R M S E=\sqrt{\sum_{i=1}^{n} \frac{\left(x_{\text {average }, i}-x_{\text {measured }, i}\right)^{2}+\left(y_{\text {average }, i}-y_{\text {measured }, i}\right)^{2}}{n}},(1) .
$$

On average, the tracked location of the mosquito deviated by 7.86 pixels (Fig. S2) from the mean of the locations measured by all 3 experimenters. This corresponds to $43.9 \%$ of the body length of an average-sized mosquito landed at the bottom of the arena (17.88 \pm 0.47 pixels or $4.87 \pm 0.12 \mathrm{~mm}$ ). In this context, and to minimize the influence of errors introduced during the digitization process, changes in velocity and angular velocity were calculated after smoothing the raw data using a low-pass butterworth filter (pass.filt(), R package $d p l R$ 1.7.2 (Bunn, 2008)) with a cut-off frequency of 0.25 (Walker, 1998). The four response variables thus quantified were visualized as a function of the mosquitoes' distance from the point of stimulus introduction (Fig. $5 \mathrm{~A}-\mathrm{D})$ and the angle of stimulus introduction (Fig. 5E-H) with a trendline $( \pm 95 \%$ confidence interval) from a single-term local regression model fit summarizing the relationship $($ geom_smooth $($ method $=$ 'loess'), R package ggplot2 3.3.5 (Wickham, 2009)).

\section{Results}

\section{Extracting visual features of a human swat}

Video tracking of a human slapping hand showed an average duration of $335 \pm 82 \mathrm{~ms}$ with an exponentially increasing expansion rate peaking at 874.9 degrees. $\mathrm{sec}^{-1}$ before covering the entire field of view of the camera ( $n=10$; Fig. 1). Side view recordings of 10 slaps, showed that the peak instantaneous velocity of the slap was of $8.01 \pm 0.66 \mathrm{~m} . \mathrm{s}^{-1}$, which is slower, but within the same order of magnitude as the recorded speed of Olympic boxers' punches (i.e., $\sim 9.14 \pm 2 \mathrm{~m} \cdot \mathrm{sec}^{-1}$; (Walilko et al., 2005)). The duration of the swat was used to program a dark looming square displayed to the mosquitoes on either a LCD monitor or on a programmable LED arena (Fig. 2A and 3A, respectively).

\section{Visual motion reduces feeding, but not landing, on a warm source of blood}

To analyze whether visual, threat-like stimuli could impact mosquitoes' ability to blood- 
observed over the course of the experiment. When looming squares were repeatedly presented to the mosquitoes, a total of 1042 landings were observed over the course of the experiment and, although there was a tendency for mosquitoes to land more during the early phase of the uniform background experiment, the difference between the two experimental conditions was not significant (Generalized linear model: $p=0.51$; Fig. 2B).

Concerning blood-feedings, on average, $69.6 \pm 2.16 \%$ of mosquitoes fed to repletion in front of the green background (Fig. 2C). However, this proportion was significantly reduced when expanding squares were introduced at regular intervals $(38.7 \pm 3.19 \%$; Generalized linear model: $p<0.001$; Fig. 2C).

Mosquitoes' response to visual threats is a function of the distance and direction of the stimulus

To better understand the role of looming visual objects in the context of threat avoidance, mosquitoes were stimulated at regular intervals, with the expanding stimulus being introduced at random positions around the arena. Because mosquitoes were free to fly (or not), this randomization led to a large number of randomized directions of stimulus introduction relative to the mosquito, and random distances between the location of the mosquito and the point of stimulus introduction on the arena (Fig. 3B-G). Mosquitoes' responses to expanding stimuli introduced at all the directions were sampled homogeneously with stimulations right behind the mosquito (<40 degrees) being sampled relatively more than the rest (Supplementary table 1). Likewise, the mosquitoes' responses as a function of their distance to the expanding stimuli was sampled homogeneously except for positions where the mosquitoes were either too close $(<100$ pixels or $27.2 \mathrm{~mm}$ in distance) to or too far (>550 pixels or $149.8 \mathrm{~mm}$ in distance) from the stimuli (Supplementary table 2).

In the absence of any stimulation, the probability of take-offs was 0.02 (Fig. 3B,E). This number significantly increased to 0.26 when an expanding stimulus was introduced (including stimuli delivered from all directions and distances; Binomial Exact test: $p<0.001)$. However, mosquitoes' escape probability varied significantly as a function of their distance to the stimulus, with peaks for stimuli delivered between 150-200 pixels away from the mosquito (40.8 - 54.5

$314 \mathrm{~mm}$; escape probability $=0.47)$ (Generalized linear model, $p<0.001)$. While the effect of the 
Model, $p=0.0817$ ), the probability of escape tented to peak at angles comprised between $80^{\circ}$ and $130^{\circ}$, i.e., laterally to the mosquito.

To confirm that mosquitoes were indeed evading the expanding square, we compared the spatial occupancy of the arena between mosquitoes that were in flight but not stimulated and mosquitoes that took off following stimulus introduction. Because of the randomization of the stimulus introduction, data were rotated to align all stimuli introductions at $180^{\circ}$. While nonstimulated mosquitoes avoided the center of the arena, they explored the entire periphery of the device (Kuiper's one sample test of uniformity: n.s.; test statistic $=1.4514$, critical value at $\alpha=$ 0.05: 1.747; Fig. 4A). On the other hand, stimulated mosquitoes not only avoided the center of the arena, but also flew to the sectors directly opposed from where the stimulus was introduced (Kuiper's one sample test of uniformity: $\mathrm{p}<0.05$; test statistic $=4.4521$ critical value at $\alpha=$ 0.05: 1.747; Fig. 4B). To further characterize the escape response displayed by mosquitoes, we analyzed how far mosquitoes would go from the point of stimulus introduction (displacement) and their overall escape direction as a function of both the distance and angle of stimulus introduction. Although there was a tendency for mosquitoes to go further away when the stimulus was introduced closer to them (blue vectors in Fig. 4C), the distance to the point of stimulus introduction had no significant effect on the displacement after take-off, regardless of the angle at which the stimulus was introduced (Fig. 4C). However, the angle of stimulus introduction had a significant effect on the direction of escape (Generalized Linear Model; $p<$ 0.001). When the stimulus was introduced in front of the mosquitoes (closer to $180^{\circ}$, red points), Mosquitoes that had stimuli introduced laterally (around 90-135 ; yellow-orange points) escaped with a wider angle $\left(>90^{\circ}\right.$ relative to the origin of the stimulus). Finally, mosquitoes that had stimuli introduced from behind $\left(<90^{\circ}\right.$, blue points) escaped by orienting the most away from the origin of the stimuli (Fig. 4D).

Analyzing four aspects of the escape response (i.e., velocity, angular velocity, displacement, and direction of escape) we found that all but the direction of escape were significantly influenced by the distance to the point of stimulus introduction (Generalized Linear Models: $p=0.003, p=0.032, p<0.001$, and $p=0.739$ for the velocity, angular velocity, 
347 the angle of stimulus introduction, only the direction of escape was significantly affected

348 (Generalized Linear Models: $p=0.82, p=0.31, p=0.59$ and $p<0.001$ for the velocity, angular 349 velocity, displacement, and direction of escape, respectively. Fig. 5E-H). In Fig. 5H, an angle of $350180^{\circ}$ indicates the front of the mosquito and $0^{\circ}$ indicates behind the mosquito, and escape directions of $180^{\circ}$ indicate flying towards the stimulus and $0^{\circ}$ away from the stimulus. These results show that mosquitoes escaped by flying the most away from the looming stimulus when it was introduced between $45^{\circ}$ and $90^{\circ}$ or from the back side of the mosquito. When the stimulus was introduced in front of the mosquitoes, they flew towards it but at a slight angle. Remarkably, take-offs were observed even for stimulus introductions immediately behind the mosquito $\left(0-45^{\circ}\right.$ angles), highlighting mosquitoes' capacity to detect threats coming from all directions along the azimuth.

\section{Discussion}

Our results demonstrate that mosquitoes can use isolated visual cues to detect and respond to potential threats, i.e., even in the absence of other sensory information (e.g., air displacement due to the host's movements). In our feeding assays, we found that the presence of looming visual cues did not impact the number of landings on the artificial feeder. However, the proportion of mosquitoes that fed to repletion was reduced by close to $50 \%$. This indicates that while the presence of rapidly moving objects in the visual field of flying mosquitoes does not impair their ability to navigate towards a source of food, once landed these same visual objects trigger an escape response. Although the identity of each mosquito wasn't maintained in our analysis, the large number of landings relative to the number of feeding events suggests that several landing attempts were made by the same mosquitoes and that several mosquitoes landed (several times) but did not feed.

Using an LED-based flight arena, we were able to further characterize this response by introducing expanding squares at randomized distances and directions relative to the focal mosquito. Results confirmed that looming squares trigger mosquitoes to take-off at a higher probability than chance (i.e., compared to the take-off probability of landed mosquitoes randomly sampled in the absence of visual stimulation: 0.02$)$. The peak escape probability (0.47) observed here was comparable to the frequency of successful escapes from a mechanical swatter by in-flight Ae. aegypti females (Cribellier, 2021). The closer to the mosquito the stimulus was 
introduced, the more likely it was to take-off and although all directions of introduction induced take-offs, threats originating laterally tended to elicit more escapes. In particular, mosquitoes were most responsive to looming stimuli that came from approximately $30^{\circ}, 90^{\circ}$, and $130^{\circ}$. This raises the question of whether the heterogeneity of the mosquito retina (Hu et al., 2014) underlies spatial specialization for the detection of threats and warrants further studies to determine the range of elevations above the plane of the substrate at which mosquitoes can detect threats. This is particularly relevant as, here, only mosquitoes that were landed horizontally on the substrate were tested. The field of view available to a mosquito actively engaged in blood-feeding may differ from its resting position (e.g., different head angle, substrate orientation). In tethered Drosophila melanogaster, the expansion of centrally versus laterally positioned objects elicited different response profiles as well, where frontal objects induced strong leg and wing-beat frequency responses but minimal changes in wing-beat amplitudes while lateral objects elicited stronger changes in wing-beat amplitude and transient increases in wing-beat frequency, but did not evoke leg responses (Tammero and Dickinson, 2002; Tammero et al., 2004).

Remarkably, the escape responses we observed were not random: after taking-off, mosquitoes rapidly maneuvered to position themselves out of the line of interception with the trajectory of the fictive threat. Analysis of the trajectories displayed after take-off shows that the direction of escape is indeed directly influenced by the angle of stimulus introduction. A similar behavior had been observed in flight in D. melanogaster where looming targets triggered visually-directed banked turns that reoriented the fly's path within a handful of wingbeats (Muijres et al., 2014). Given the high wing-beat frequency of mosquitoes $(400-500 \mathrm{~Hz})$, highspeed recordings will be required to quantify the fine scale adjustments performed by mosquitoes to take-off and reorient within such a short timeframe. Additionally, the closer the mosquito was from the point of introduction of the threat, the faster and further they escaped by following a less tortuous path (indicated by a lower angular velocity). These results open new avenues for investigating distance perception by mosquitoes.

It is possible that the response probability to the looming stimuli was actually higher than what we observed by only quantifying take-offs as it may have taken other forms, such as a freeze response to threats. Indeed, other animals have been shown to make a decision to either

407 freeze or flee when threat is presented (Yilmaz and Meister, 2013). Our analysis focused on 408 fleeing responses, given that freezing responses could not be identified and characterized 
409

410

411

412

413

414

415

416

417

418

419

420

421

422

423

424

425

426

427

428

429

430

431

432

433

434

435

436

437

438

439

because we focused on landed mosquitoes. In addition, because of the size of the clear acrylic cage the mosquitoes were contained in, escape responses could only be examined over a short period of time, as mosquitoes would rapidly reach the wall of the container and redirect their flight trajectory. However, in spite of this limitation and while quantifying responses in 2dimensions only, results of the present study show clear visual responses to isolated visual stimuli, warranting further 3-dimensional analysis in larger scale (e.g., wind tunnel) assays.

The most striking feature of the escape response quantified here is the tight relationship between the angle at which the stimulus is introduced and the direction of escape. Within $2 / 3 \mathrm{rd}$ of a second, mosquitoes did not just take off and scatter away from the stimulus. Conversely, they displayed a clear response pattern that can be preliminarily divided into three categories: 1) when the visual threats appear towards the front of the mosquito, they take off and veer at angle between 45 and $90^{\circ}$ away from the origin of the threat; 2) when visual threats appear laterally, mosquitoes take off and re-orient the most away from the threat; 3) when visual threats appear from behind the mosquitoes, they take off and turn slightly, as needed in order to get away from the line of interception.

Altogether, results from the present study provide some insights into the "pre-biting' or "pre-attack" resting behavior observed in several anopheline and culicine mosquito species (Tuno et al., 2003). Because this "pre-biting" rest was more frequently observed at higher mosquito densities, we had emitted the hypothesis that mosquitoes possessed the ability to evaluate the level of defensiveness from afar, possibly using vision in this context. In this hypothetical scenario, mosquitoes would land near the host and wait for its defensive behaviors to decrease before approaching, thus increasing the probability of a successful blood-feeding. However, in our artificial feeding assays, the number of landings on a host mimic was not affected by visual threats, only the proportion of successful feedings on an otherwise defenseless feeder. This differs from other studies conducted in tabanid flies, where stripes present on the fur of the host made it much more difficult for the flies to land on them (Caro et al., 2019). As a result, horses with solid color experienced more fly landings.

In nature, another critical contributor to mosquitoes' feeding success is the density of female mosquitoes around the host (Day and Edman, 1984; Edman et al., 1972; Edman et al., 1974; Walker and Edman, 1985). Indeed, as the density of mosquitoes increases so does the level of defensiveness of the host, which negatively correlates with the proportion of females that 
successfully blood-feed (Edman et al., 1972). As a consequence, mosquitoes prefer to feed on the least defensive or least active host over more defensive ones (Edman et al., 1974; Warnes and Finlayson, 1987). While our results suggest that Ae. aegypti gauges the defensiveness of a host only after landing on it, it is worth highlighting that only visual cues were available in our experiments. But while mechanical cues (i.e., air displacement) generated by a mammal tail simulator significantly prevented mosquitoes from landing (Matherne et al., 2018), whether they can trigger the pre-biting resting behavior remains to be determined. In a previous study, when a mechanical vibration calibrated to mimic an average swat was repeatedly paired with host olfactory cues, Ae. aegypti females learned the association between the mechanical stimulus and the odor, and subsequently avoided the trained odor (Vinauger et al., 2018). Mechanical cues thus contribute to host selection processes (Vinauger et al., 2018; Wolff and Riffell, 2018; Wynne et al., 2020) and, by disturbing the ability of mosquitoes to land on the host, most likely contribute to a mosquito's evaluation of the host's defensiveness.

Beyond their role when presented in isolation, sensory cues from multiple modalities (e.g., vision and olfaction) are integrated by the mosquito brain (San Alberto et al., 2021; van Breugel et al., 2015; Vinauger et al., 2019). In the context of evading host defenses, Cribellier $e t$ a mechanical swatter in bright light conditions, i.e., when both visual and mechanical cues were available. Interestingly, night-active Anopheles coluzzii were most successful at escaping in the dark, suggesting that nocturnal mosquitoes maximize their escape performance by adjusting parameters of their flight behavior itself, such as adopting a more stochastic (i.e., protean) flight (Cribellier et al., 2021). In the present study, we provide an experimental paradigm that permits the presentation of visual cues in isolation from mechanical perturbations. In future work, this paradigm could be adapted to spatially decouple visual and mechanosensory cues to deepen our understanding of multimodal sensory integration processes in the context of threat avoidance.

Vision has been shown to be used by many insect species in a variety of biological 467 Spaethe et al., 2006), including identifying predators or threats. Across the animal kingdom, 468 looming stimuli are typically characterized as a threat and elicit an escape response (Peek and 469 Card, 2016). Here, we showed that isolated visual looming stimuli are sufficient to elicit an 470 escape response in Ae. aegypti mosquitoes. This highlights the importance of this historically 
overlooked sensory modality as, in addition to recent studies that characterized its role in the

472 host-seeking context (Liu and Vosshall, 2019; San Alberto et al., 2021; van Breugel et al., 2015;

473 Vinauger et al., 2019; Zhan et al., 2021), vision also contributes to the identification and

474 avoidance of potential threats. The looming stimuli used in the present study were designed to

475 mimic a human swat, as reflecting the preferred host of Ae. aegypti. However, mosquito species

476 differ in their host preference, with some species biting smaller or larger mammals (Matherne et

477 al., 2018), amphibians, or birds (Molaei et al., 2008), which vary drastically in their antiparasitic

478 and defensive behaviors. As a consequence, it is likely that the tuning for certain ranges of object

479 sizes, shapes, expansion rates, as well as linear and angular velocities will differ between

480 mosquito species. In D. melanogaster, such feature-dependent effects have been described in

481 tethered preparations where taller stripes (above $50^{\circ}$ in height) elicited fixation by the animal but

482 shorter stripes ( 8 to $37^{\circ}$ tall) triggered anti-fixation behaviors (Maimon et al., 2008). Compared

483 to flies, mosquitoes face an additional challenge as females are required to approach hosts (larger

484 and slowly expanding visual objects) that can turn into threats at any point (i.e., swat appendages

485 at high velocities). Without a blood meal, Ae. aegypti mosquitoes cannot produce progeny

486 (Christophers, 1960). Given the strong selective pressures associated with host seeking and blood

487 feeding, one might thus expect a similarly fine tuning of the response to features of visual

488 stimuli. Future work investigating this area will provide invaluable insights into the adaptations

489 underlying each species-specific mosquito-host association.

490 To conclude, results from the present study show that visual threat-like-stimuli alone are 491 sufficient to disrupt Ae. aegypti's blood feeding behavior and trigger a stereotypical escape 492 response influenced by the distance and direction of the threat. This work opens new research 493 avenues for improving our understanding of the role of vision in mosquito biology and 494 developing control strategies that target this sensory modality.

496 Acknowledgements

497 We thank Drs. Chloé Lahondère, Jake Tu, and Jake Socha for their feedback and valuable 498 insights on the experimental design and the manuscript. We also thank Danny Eanes and Steve 499 Lowe for their help with technical details, Chanel Hsu for her help with preliminary data 500 management and visualization, and Shajaesza Diggs and Darren Dougharty for their help with 501 mosquito rearing. 


\section{Competing Interests}

505 The authors declare no competing or financial interests.

\section{Funding}

508 This material is based upon work supported by the US Department of Agriculture National 509 Institute of Food and Agriculture under Hatch project \# 1017860 to C.V.

\section{Author contributions}

512 Conceptualization: N.W., K.C, C.V.; Methodology: N.W., K.C., C.V.; Software: K.C., C.V.;

513 Validation: N.W.; Formal analysis: K.C., C.V.; Investigation: N.W., L.F.; Resources: C.V.; Data

514 Curation: N.W., K.C.; Writing - original draft preparation: N.W., K.C.; Writing - review and 515 editing: N.W., K.C., C.V.; Visualization: N.W., K.C., C.V.; Supervision: C.V.; Project 516 administration: N.W., C.V.; Funding acquisition: C.V.

\section{Data Availability}

519 Data will be made available from the Dryad Digital Repository (DOI will be inserted here and 520 reference will be added to the list of reference) upon manuscript acceptance.

\section{References}

Bates, D., Mächler, M., Bolker, B. and Walker, S. (2014). Fitting Linear Mixed-Effects

Bunn, A. G. (2008). A dendrochronology program library in R (dplR). Dendrochronologia 26, Models using lme4. arXiv [stat.CO]. $115-124$.

Caro, T., Argueta, Y., Briolat, E. S., Bruggink, J., Kasprowsky, M., Lake, J., Mitchell, M. J., Richardson, S. and How, M. (2019). Benefits of zebra stripes: Behaviour of tabanid flies around zebras and horses. PLoS One 14, e0210831.

530 Christophers, S. R. (1960). Aedes aegypti: the yellow fever mosquito. (ed. CUP Archive).

531 Clements, A. N. (1999). The biology of mosquitoes. Volume 2: sensory reception and behaviour. 
CABI publishing.

Cribellier, A. (2021). Biomechanics of flying mosquitoes during capture and escape (Doctoral dissertation, Wageningen University).

Cribellier, A., Spitzen, J., Straw, A. D., van Leeuwen, J. L. and Muijres, F. T. (2021). Escape flight performances of night-active malaria mosquitoes: the role of visual and airflow cues of an approaching object. In Integrative and Comparative Biology, pp. E170-E171. OXFORD UNIV PRESS INC JOURNALS DEPT, 2001 EVANS RD, CARY, NC 27513 USA.

Day, J. F. and Edman, J. D. (1984). Mosquito engorgement on normally defensive hosts depends on host activity Patterns. J. Med. Entomol. 21, 732-740.

Edman, J. D., Webber, L. A. and Kale, H. W. (1972). Effect of mosquito density on the interrelationship of host behavior and mosquito feeding success. Am. J. Trop. Med. Hyg. 21, 487-491.

Edman, J. D., Webber, L. A. and Schmid, A. A. (1974). Effect of host defenses on the feeding pattern of Culex nigripalpus when offered a choice of blood sources. J. Parasitol. 60, 874-883.

Eilerts, D. F., VanderGiessen, M., Bose, E. A., Broxton, K. and Vinauger, C. (2018). Odorspecific daily rhythms in the olfactory sensitivity and behavior of Aedes aegypti mosquitoes. Insects 9, 147.

Grant, A. J. and O'Connell, R. J. (2007). Age-related changes in female mosquito carbon dioxide detection. J. Med. Entomol. 44, 617-623.

Hothorn, T., Bretz, F. and Westfall, P. (2008). Simultaneous inference in general parametric models. Biom. J. 50, 346-363.

Hu, X., Leming, M. T., Whaley, M. A. and O'Tousa, J. E. (2014). Rhodopsin coexpression in UV photoreceptors of Aedes aegypti and Anopheles gambiae mosquitoes. J. Exp. Biol. 217, 1003-1008.

Liu, M. Z. and Vosshall, L. B. (2019). General visual and contingent thermal cues interact to elicit attraction in female Aedes aegypti mosquitoes. Curr. Biol. 29, 2250-2257.e4.

Maimon, G., Straw, A. D. and Dickinson, M. H. (2008). A simple vision-based algorithm for decision making in flying Drosophila. Curr. Biol. 18, 464-470.

Matherne, M. E., Cockerill, K., Zhou, Y., Bellamkonda, M. and Hu, D. L. (2018). Mammals repel mosquitoes with their tails. J. Exp. Biol. 221,.

Molaei, G., Andreadis, T. G., Armstrong, P. M. and Diuk-Wasser, M. (2008). Host-feeding patterns of potential mosquito vectors in Connecticut, USA: molecular analysis of bloodmeals from 23 species of Aedes, Anopheles, Culex, Coquillettidia, Psorophora, and Uranotaenia. J. Med. Entomol. 45, 1143-1151. 
Muijres, F. T., Elzinga, M. J., Melis, J. M. and Dickinson, M. H. (2014). Flies evade looming targets by executing rapid visually directed banked turns. Science 344, 172-177.

Olberg, R. M. (2012). Visual control of prey-capture flight in dragonflies. Curr. Opin. Neurobiol. 22, 267-271.

Palavalli-Nettimi, R. and Theobald, J. C. (2020). Small eyes in dim light: Implications to spatio-temporal visual abilities in Drosophila melanogaster. Vision Res. 169, 33-40.

Palavalli-Nettimi, R., Ogawa, Y., Ryan, L. A., Hart, N. S. and Narendra, A. (2019). Miniaturisation reduces contrast sensitivity and spatial resolving power in ants. J. Exp. Biol. 222,

Peek, M. Y. and Card, G. M. (2016). Comparative approaches to escape. Curr. Opin. Neurobiol. 41, 167-173.

Peirce, J., Gray, J. R., Simpson, S., MacAskill, M., Höchenberger, R., Sogo, H., Kastman, E. and Lindeløv, J. K. (2019). PsychoPy2: Experiments in behavior made easy. Behav. Res. Methods 51, 195-203.

Ponlawat, A. and Harrington, L. C. (2005). Blood Feeding Patterns of Aedes aegypti and Aedes albopictus in Thailand. J. Med. Entomol. 42, 844-849.

Reid, J. A. (1968). Anopheline mosquitoes of Malaya and Borneo. Studies from the Institute for Medical Research, Malaysia. Anopheline mosquitoes of Malaya and Borneo. Studies from the Institute for Medical Research, Malaysia.

Reiser, M. B. and Dickinson, M. H. (2008). A modular display system for insect behavioral neuroscience. J. Neurosci. Methods 167, 127-139.

San Alberto, D. A., Rusch, C., Zhan, Y., Straw, A. D. and Montell, C. (2021). The olfactory gating of visual preferences to human skin and colors in mosquitoes. bioRxiv.

Spaethe, J., Chittka, L. and Skorupski, P. (2006). Visual search and decision making in bees: time, speed and accuracy. Int. J. Comp. Psychol.

Tallon, A. K., Hill, S. R. and Ignell, R. (2019). Sex and age modulate antennal chemosensoryrelated genes linked to the onset of host seeking in the yellow-fever mosquito, Aedes aegypti. Sci. Rep. 9, 43.

Tammero, L. F. and Dickinson, M. H. (2002). Collision-avoidance and landing responses are mediated by separate pathways in the fruit fly, Drosophila melanogaster. J. Exp. Biol. 205, 2785-2798.

Tammero, L. F., Frye, M. A. and Dickinson, M. H. (2004). Spatial organization of visuomotor reflexes in Drosophila. J. Exp. Biol. 207, 113-122.

Taylor, B. and Jones, M. D. (1969). The circadian rhythm of flight activity in the mosquito Aedes aegypti (L.). The phase-setting effects of light-on and light-off. J. Exp. Biol. 51, 
59-70.

Tuno, N., Tsuda, Y., Takagi, M. and Swonkerd, W. (2003). Pre- and postprandial mosquito resting behavior around cattle hosts. J. Am. Mosq. Control Assoc. 19, 211-219.

Tuno, N., Tsuda, Y. and Takagi, M. (2017). How zoophilic japanese encephalitis vector mosquitoes feed on humans. J. Med. Entomol. 54, 8-13.

van Breugel, F., Riffell, J., Fairhall, A. and Dickinson, M. H. (2015). Mosquitoes Use Vision to Associate Odor Plumes with Thermal Targets. Curr. Biol. 25, 2123-2129.

Vinauger, C., Lahondère, C., Wolff, G. H., Locke, L. T., Liaw, J. E., Parrish, J. Z., Akbari, O. S., Dickinson, M. H. and Riffell, J. A. (2018). Modulation of Host Learning in Aedes aegypti Mosquitoes. Curr. Biol. 28, 333-344.e8. L., Akbari, O. S. and Riffell, J. A. (2019). Visual-olfactory integration in the human disease vector mosquito, Aedes aegypti. Curr. Biol. 29, 2509-2516.e5.

Walilko, T. J., Viano, D. C. and Bir, C. A. (2005). Biomechanics of the head for Olympic boxer punches to the face. Br. J. Sports Med. 39, 710-719.

Walker, J. A. (1998). Estimating velocities and accelerations of animal locomotion: a simulation experiment comparing numerical differentiation algorithms. J. Exp. Biol. 201, 981-995.

Walker, E. D. and Edman, J. D. (1985). The Influence of Host Defensive Behavior on Mosquito (Diptera: Culicidae) Biting Persistence1. J. Med. Entomol. 22, 370-372.

Warnes, M. L. and Finlayson, L. H. (1987). Effect of host behaviour on host preference in Stomoxys calcitrans. Med. Vet. Entomol. 1, 53-57.

Wickham, H. (2009). ggplot2: Elegant Graphics for Data Analysis. Springer, New York, NY.

Wolff, G. H. and Riffell, J. A. (2018). Olfaction, experience and neural mechanisms underlying mosquito host preference. J. Exp. Biol. 221,.

627 World Health Organization (2018). World Health Statistics 2018.

Wynne, N. E., Lorenzo, M. G. and Vinauger, C. (2020). Mechanism and plasticity of vectors' host-seeking behavior. Curr Opin Insect Sci 40, 1-5.

Yilmaz, M. and Meister, M. (2013). Rapid innate defensive responses of mice to looming visual stimuli. Curr. Biol. 23, 2011-2015. 
bioRxiv preprint doi: https://doi.org/10.1101/2022.01.08.475512; this version posted January 10, 2022. The copyright holder for this preprint (which was not certified by peer review) is the author/funder. All rights reserved. No reuse allowed without permission.

636 


\section{Figure Legends}

638

639
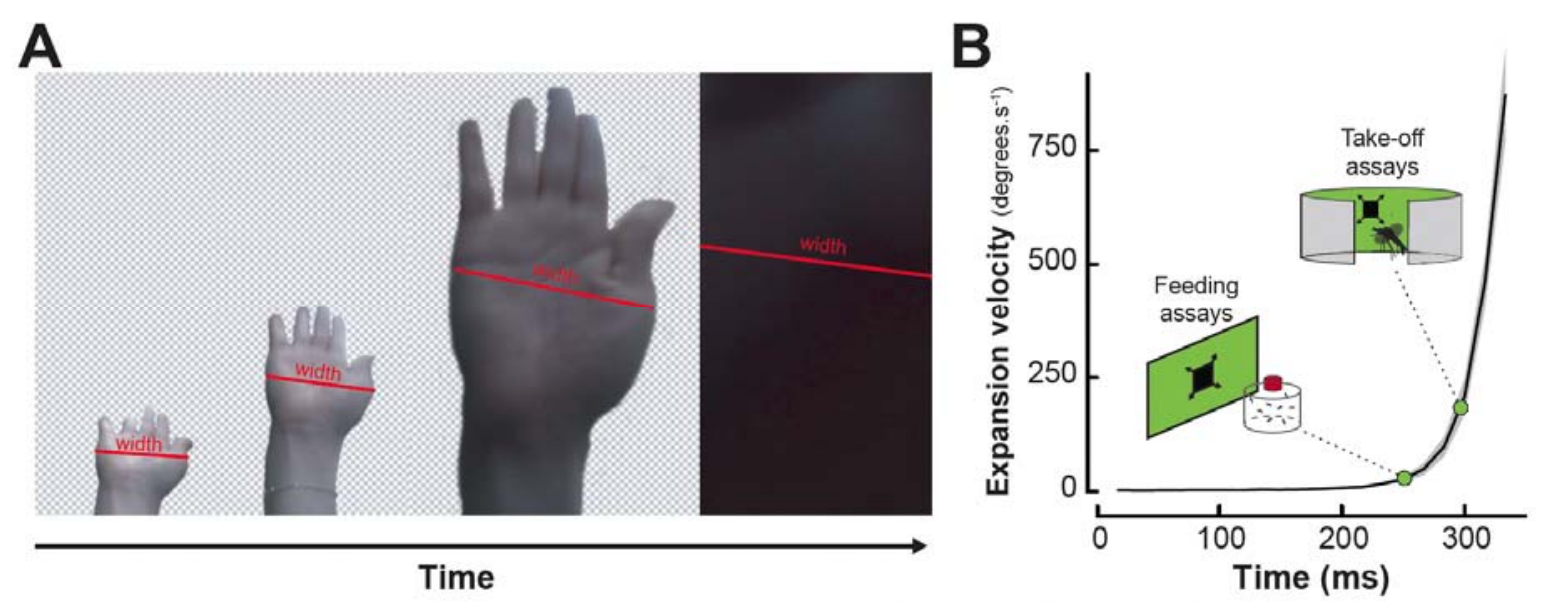

641 Figure 1. Developing an artificial visual swat.

642 (A) Representative screenshots from the video recording of hand slaps filmed at 60 frames per second. The red lines illustrate the measurement of the widest part of the hand. Front view recordings were paired with side-view recordings used to quantify the linear velocity of the hand (data not shown). (B) Mean expansion velocity (in degrees per second) of a hand swat as a function of time (black line; $\mathrm{n}=10$ replicates). Grey shaded area indicates the standard error to the mean expansion velocity. Green points indicate the expansion velocities used to program the expanding squares displayed in the feeding and take-off assays, respectively. 
A
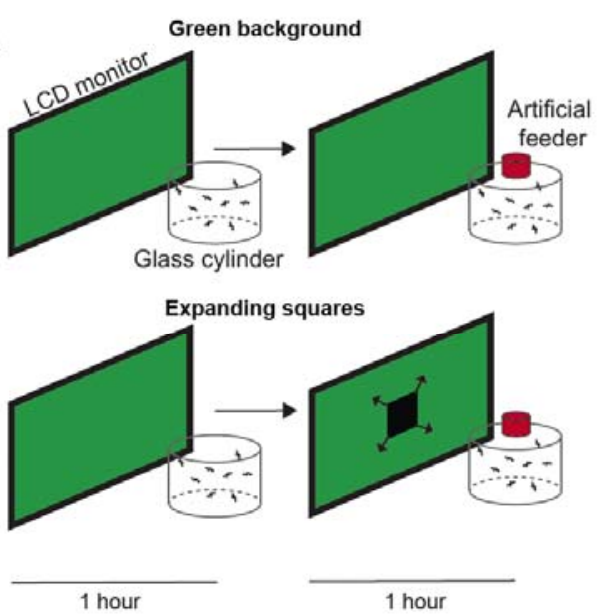

653

654

655

656

657

658

659

660

661

662

663

664

665
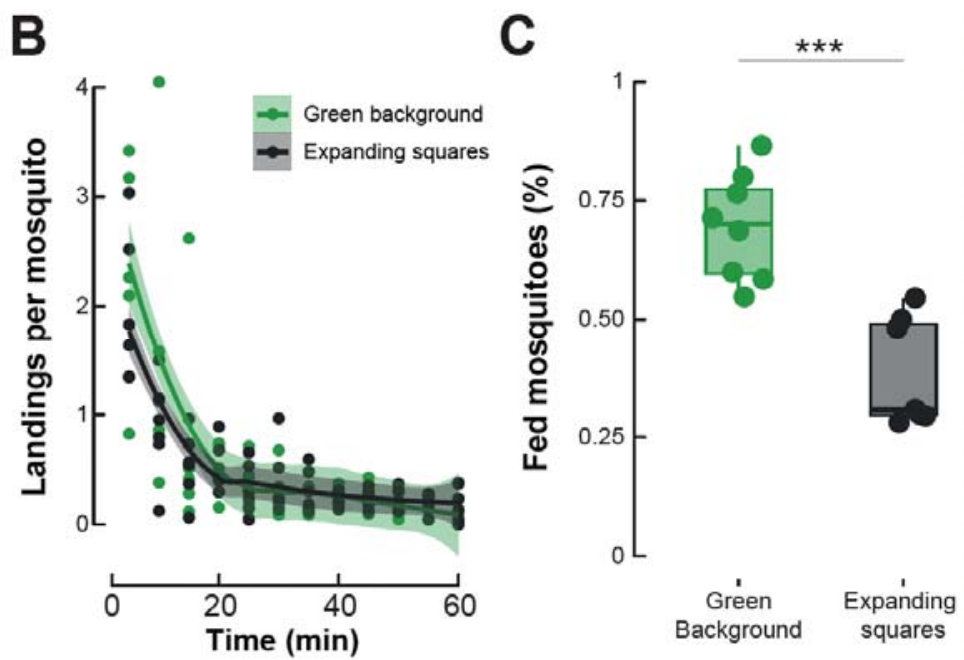

Figure 2. Landing and feeding on a host mimic in different visual contexts.

(A) Schematic of the experimental apparatus. Groups of mosquitoes are enclosed in a glass cylinder positioned in front of an LCD monitor. To acclimate to the experimental conditions, mosquitoes are left unperturbed in front of a green background for 1 hour. Control groups (top) were exposed to a second hour of green background during which the number of landing and feedings on a membrane feeder were quantified. Treatment groups (bottom) were exposed to randomly positioned squares that symmetrically expand at periodic intervals. (B) Number of landings per mosquito quantified every five minutes of the second hour of the experiment for the control (green) and treatment groups (dark grey). (C) Proportion of feedings quantified at the end of the second hour of the experiment for the control (green, $\mathrm{n}=8$ ) and treatment groups (dark grey, $\mathrm{n}=7$ ). Asterisks denote significant differences (***: $p<0.001$; Generalized linear model). 
A

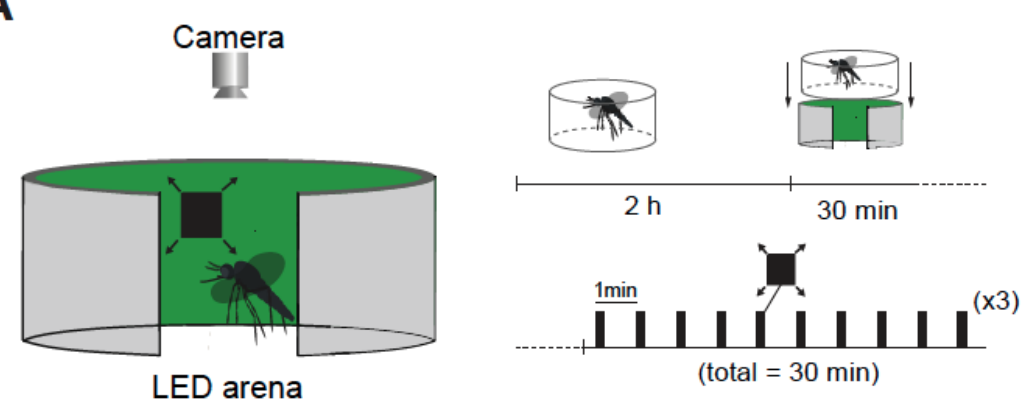

B

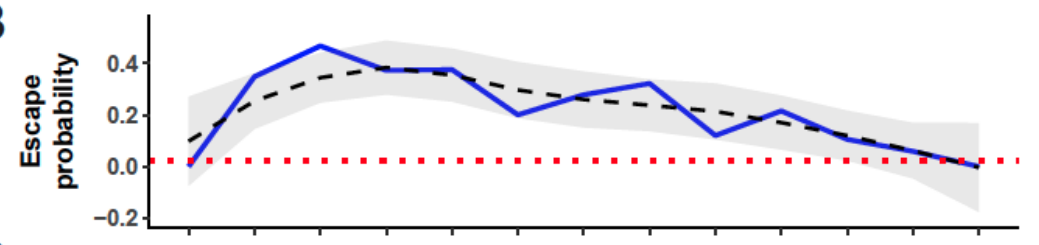

C
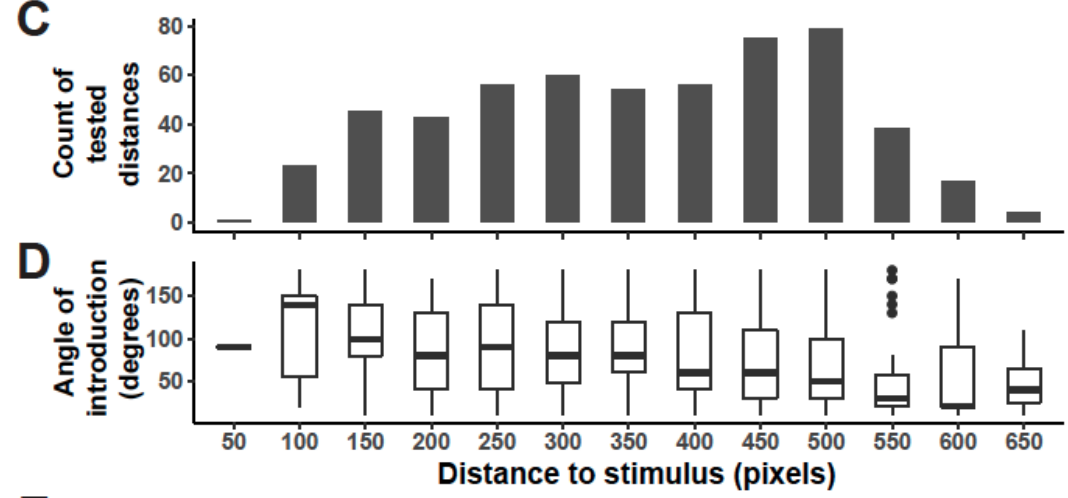

E

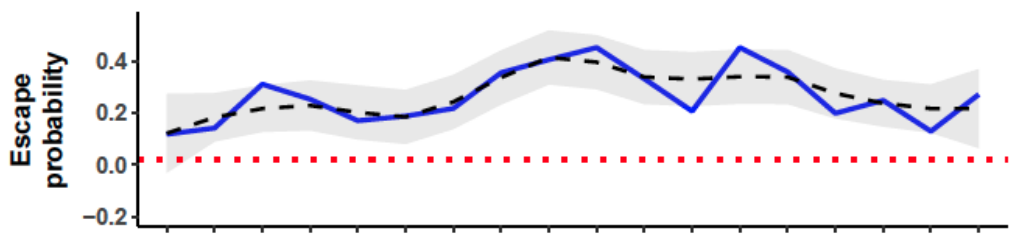

F
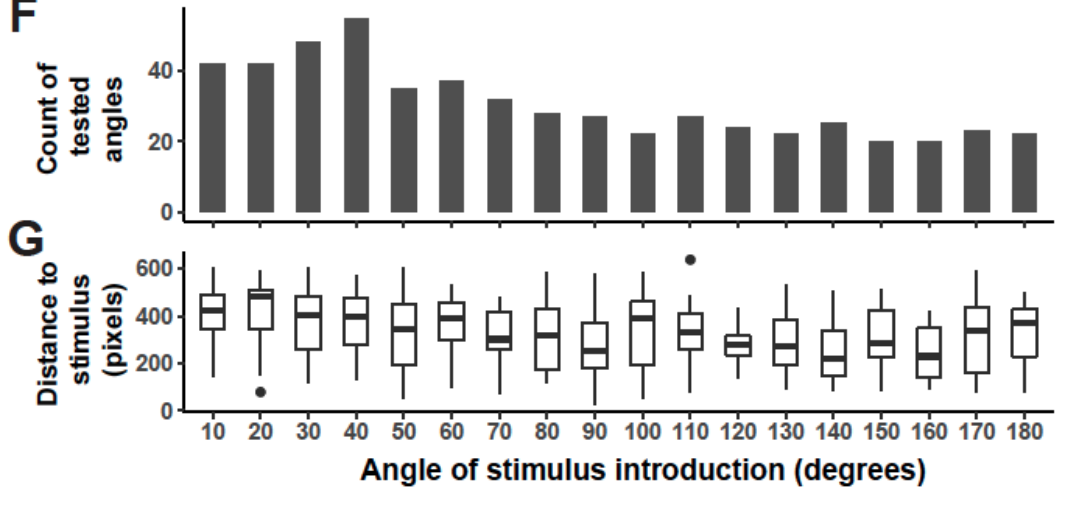

Figure 3. Escape probability as a function of the distance and direction of the stimulus.

(A) Schematic of the experimental apparatus (left) and graphical representation of the experiment's timeline (right) where an individual female mosquito is isolated in a clear cylinder 
672 mosquito is provided 30 minutes to acclimatize to the conditions before being stimulated with a 673 looming square every minute for 30 minutes. (B,E) Mosquitoes' probability to escape (take-off) 674 after introduction of the expanding stimuli, represented as a function of the distance to the point 675 of stimulus introduction (B) or as a function of the direction of stimulus introduction (E). A 676 trendline (dashed, black) from a single-term linear model fit with 95\% confidence interval (gray) 677 summarizes the relationship between escape probability and the predictor variables. The dotted 678 horizontal line (red) denotes the baseline escape probability (take-off) in the absence of any 679 expanding stimuli. (C,F) Number of experimental trials. (D,G) Distribution of the direction of 680 stimuli introduction (degrees). In (B-D), the response variables are visualized as a function of the 681 mosquitoes' distance from the point of stimulus introduction (0-650 pixels or $0-177.14 \mathrm{~mm}$ ) 682 represented as a categorical variable, i.e., 50 pixels per category. In (E-G), the response variables 683 are visualized as a function of the angle of stimulus introduction (0-180 degrees, where 0 degrees 684 is behind and 180 in front of the mosquito) represented as a categorical variable, i.e., 10 degrees 685 per category. 
A

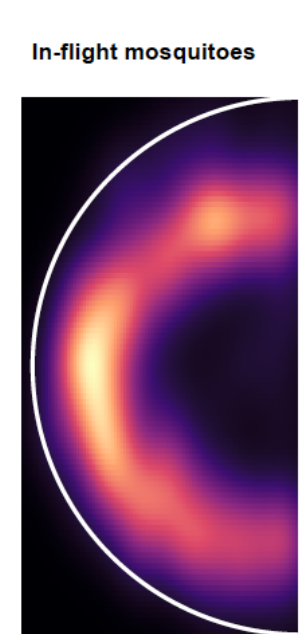

C

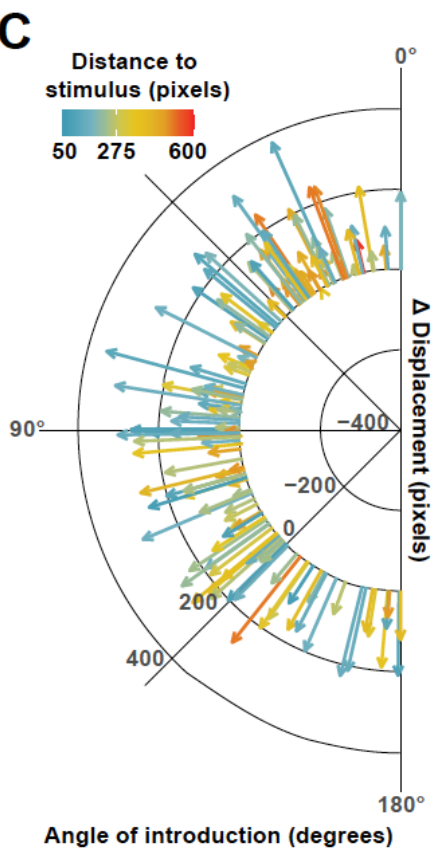

B

Stimulated mosquitoes

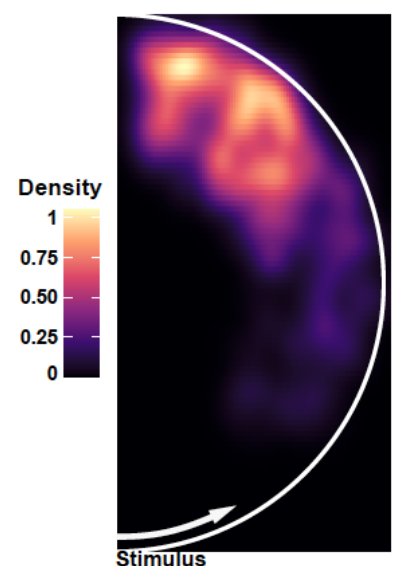

D
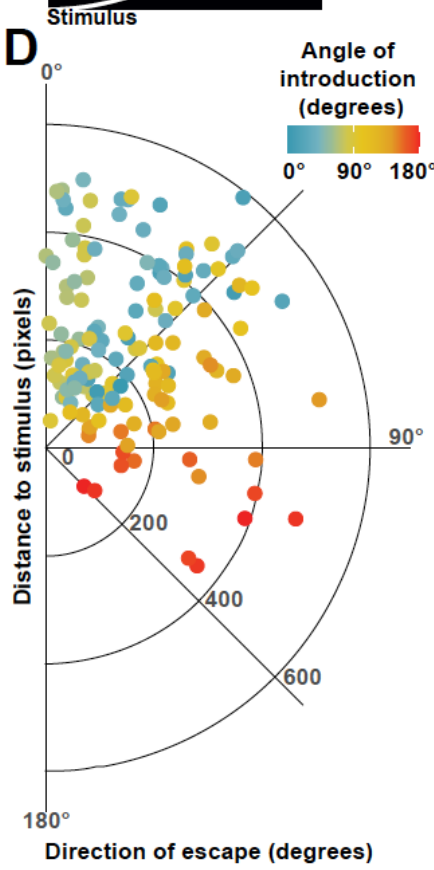

\section{Figure 4. Overall behavioral responses to the looming square.} mirrored on the vertical axis; the white semi circles indicate the relative position of the arena's walls. (A) Normalized histogram of the location of mosquitoes during randomly selected flight bouts, without visual stimulation. (B) Normalized histogram of the location of mosquitoes during the first second after a take-off triggered by the introduction of a looming square. Data are oriented such as the stimulus introduction (represented as a white arrow) is positioned at the bottom of the figure. (C) Circle plot showing the displacement of mosquitoes (i.e., distance travelled on a scale ranging from $0-400$ pixels or $0-109 \mathrm{~mm}$ ) within $2 / 3^{\text {rd }}$ of a second of stimulustriggered take-offs. The length of the vectors encodes for displacement, color coded as a function of the distance to the stimulus' point of introduction, and the position of the vector around the circle encodes for the direction of stimulus introduction, where $180^{\circ}$ is to the front of the mosquito and 0 behind the mosquito. Each vector represents one take-off (D) Circle plot representing the direction of escape (position around the circle, where 180 is towards and 0 away 
702 from the stimulus) as a function of the distance to the stimulus point of introduction (position 703 along the radius of the circle) and color coded as a function of the direction of stimulus 704 introduction (where 180 is to the front of the mosquito and 0 behind the mosquito). Each point 705 represents one take-off $(\mathrm{n}=142)$. 

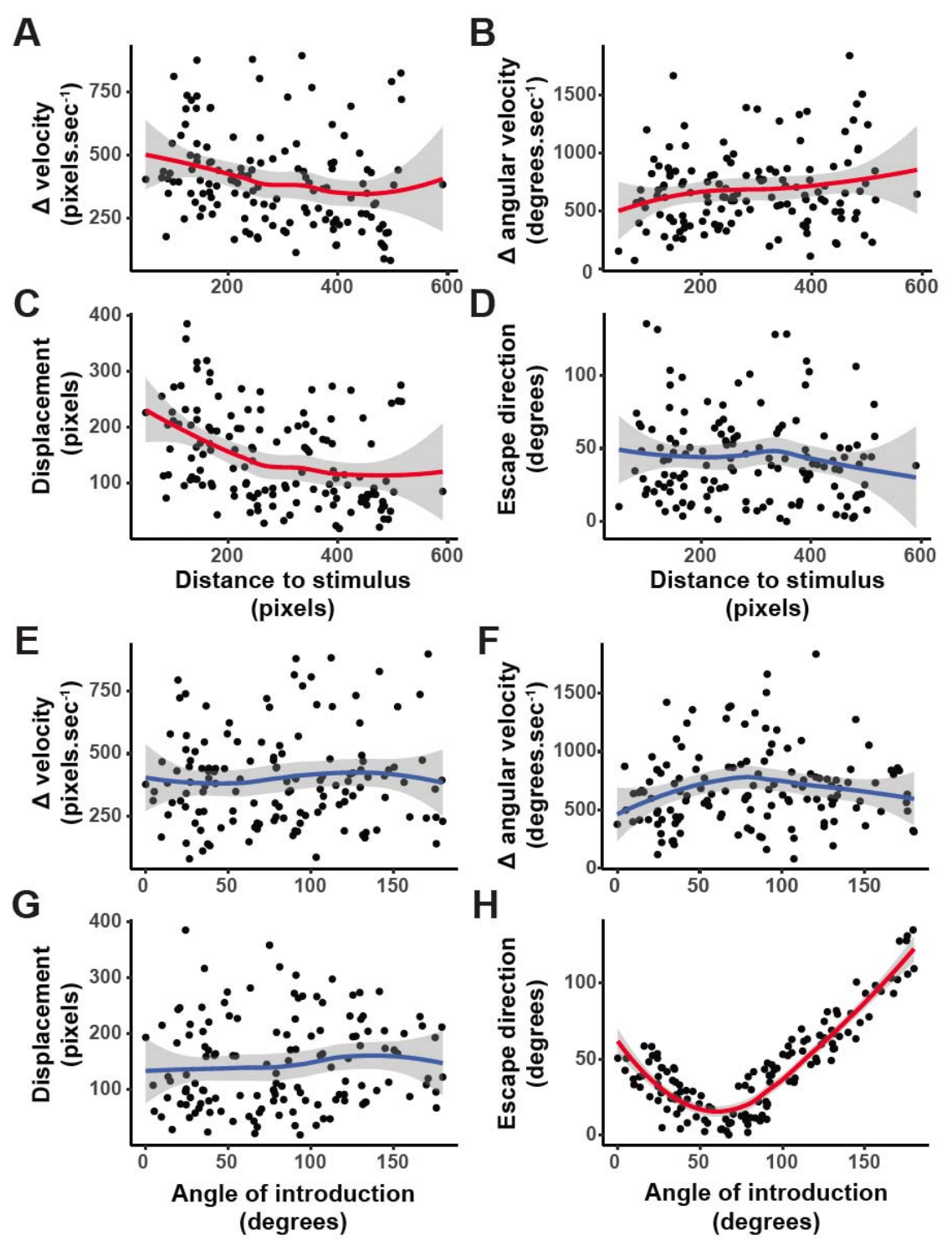

Figure 5. Statistical analysis of the escape strategy displayed by mosquitoes.

Mosquitoes' responses quantified as $(\mathbf{A}, \mathbf{E})$ Change in velocity (pixels.sec $\left.{ }^{-1}\right),(\mathbf{B}, \mathbf{F})$ Change in angular velocity (degrees. $\left.\sec ^{-1}\right),(\mathbf{C}, \mathbf{G})$ Displacement (pixels), and $(\mathbf{D}, \mathbf{H})$ Direction of escape (degrees), where 180 is directly towards the stimulus and 0 away from the stimulus. In (A-D), the response variables are visualized as a function of the mosquitoes' distance from the point of stimulus introduction (0-600 pixels or 0-163.5 $\mathrm{mm})$. In $(\mathbf{E}-\mathbf{H})$, the response variables are visualized as a function of the angle of stimulus introduction, where 180 is in front of the mosquito and 0 is behind the mosquito. In (A-H), a trend line from a single-term local regression model fit with $95 \%$ confidence interval (gray) summarizes the relationship between the response and predictor variables. Red trend lines indicate significant effects of the predictive factors (distance to stimulus or angle of stimulus introduction, respectively) on the response variables, and blue trend lines indicate non-significant effects (Generalized Linear Models, $\alpha=0.05$ ). 


\section{Supplementary Information}

724

A
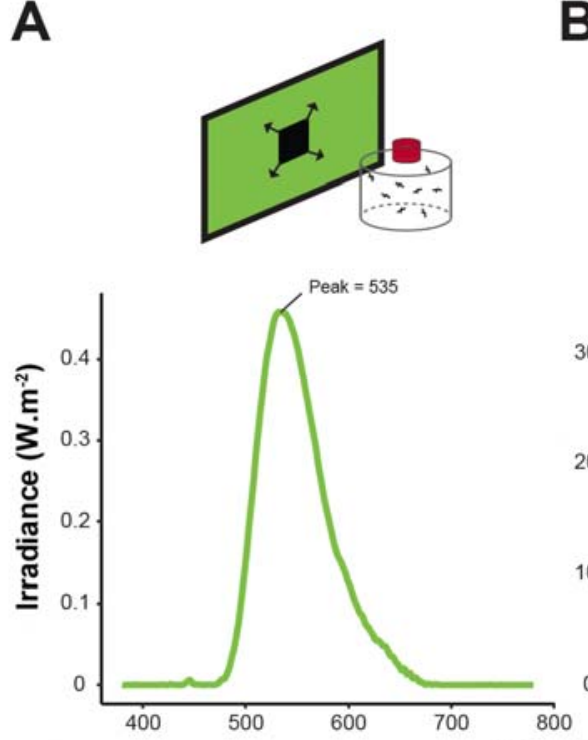

B
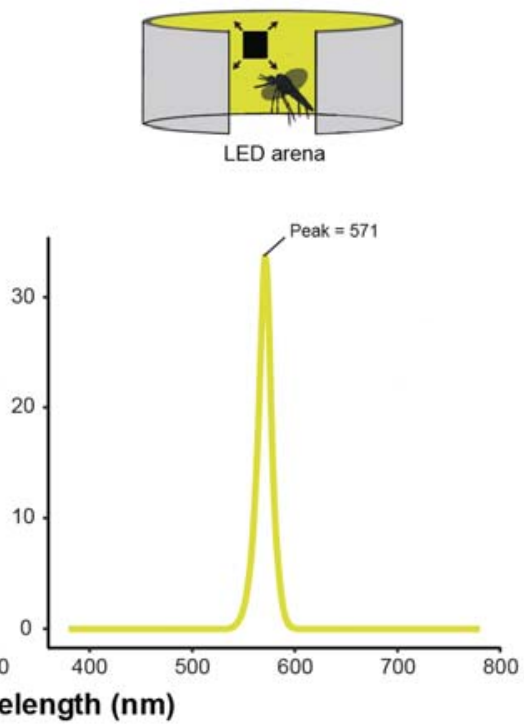

\section{Figure S1. Spectral irradiance of the visual displays.}

727 The spectral signature of the green backgrounds used in the feeding (A) and take-off (B) assays 728 was determined by measuring the irradiance of displays for wavelengths between 380 and 780 $729 \mathrm{~nm}$, using a portable spectral irradiance colorimeter (OHSP350, Hopoocolor, Hangzhou China). 

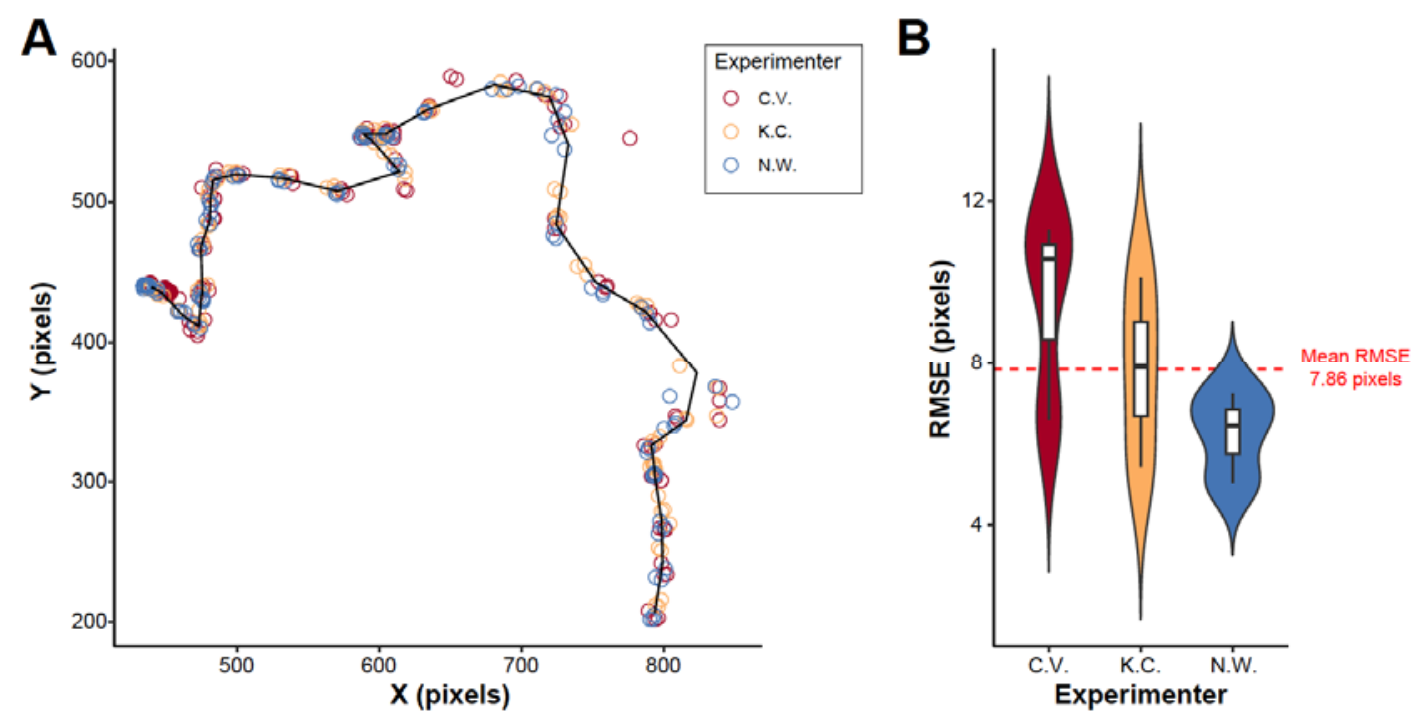

734 Figure S2. Digitization error associated with manual tracking.

735 (A) Tracking of a 59-frame flight sequence by 3 different experimenters on 3 consecutive days. Experimenters are identified by their initials. Data were collected in triplicates and results are color-coded for the individual experimenter. The solid black line indicates the mean trajectory calculated from the mean of the coordinates tracked by all 3 experimenters. (B) Digitizing error quantified as the Root Square Mean Error (RMSE), representing the average deviation from the mean trajectory, for each experimenter. The dashed red line indicates the mean RMSE. 

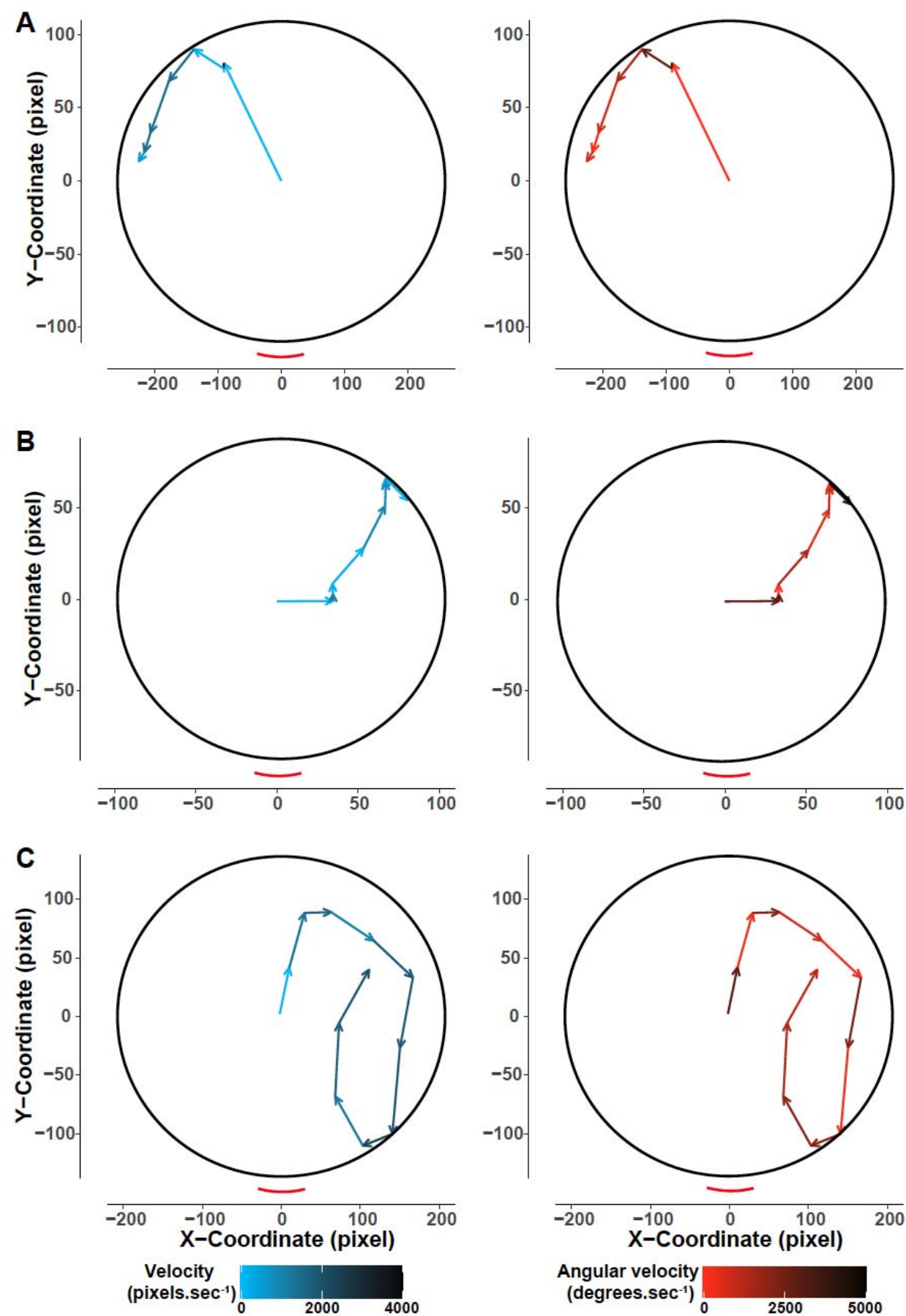

Figure S3. Representative trajectories of mosquitoes' escape (take-off) responses. expanding stimulus. The visualized cartesian coordinates are tracks of the respective mosquitoes' head 20 frames post stimulus introduction (i.e., $2 / 3$ of a second). The trajectories are rotated to fictively re-position the stimuli introductions at $180^{\circ}$. The trajectories are color coded as a function of the mosquitoes' velocity (left panel, blue) and angular velocity (right panel, red). 
Movie 1. Illustration of stimuli delivered in the two experiments.

Movie 2. Example of a mosquito taking-off in response to the looming square.

Supplementary Table 1: Number of mosquitoes sampled for their responses to expanding stimuli as a function of the angle of stimulus introduction.

\begin{tabular}{|c|c|c|c|c|}
\hline $\begin{array}{l}\text { Stimulus } \\
\text { direction } \\
\text { (degrees) }\end{array}$ & $\begin{array}{l}\text { No. } \\
\text { trials }\end{array}$ & $\begin{array}{c}\text { Lower } \\
\text { CI }\end{array}$ & $\begin{array}{c}\text { Upper } \\
\text { CI }\end{array}$ & z-ratio \\
\hline $0-10$ & 42 & 31 & 56.8 & 24.223 \\
\hline $10-20$ & 42 & 31 & 56.8 & 24.223 \\
\hline $20-30$ & 48 & 36.2 & 63.7 & 26.82 \\
\hline $30-40$ & 55 & 42.2 & 71.6 & 29.719 \\
\hline $40-50$ & 35 & 25.1 & 48.7 & 21.034 \\
\hline $50-60$ & 37 & 26.8 & 51.1 & 21.964 \\
\hline $60-70$ & 32 & 22.6 & 45.3 & 19.605 \\
\hline $70-80$ & 28 & 19.3 & 40.6 & 17.632 \\
\hline $80-90$ & 27 & 18.5 & 39.4 & 17.126 \\
\hline $90-100$ & 22 & 14.5 & 33.4 & 14.498 \\
\hline $100-110$ & 27 & 18.5 & 39.4 & 17.126 \\
\hline $110-120$ & 24 & 16.1 & 35.8 & 15.569 \\
\hline
\end{tabular}




\begin{tabular}{|l|c|c|c|c|}
\hline $210-130$ & 22 & 14.5 & 33.4 & 14.498 \\
\hline $130-140$ & 25 & 16.9 & 37 & 16.094 \\
\hline $140-150$ & 20 & 12.9 & 31 & 13.397 \\
\hline $150-160$ & 20 & 12.9 & 31 & 13.397 \\
\hline $160-170$ & 23 & 15.3 & 34.6 & 15.037 \\
\hline $170-180$ & 22 & 14.5 & 33.4 & 14.498 \\
\hline
\end{tabular}

Supplementary Table 2: Number of mosquitoes sampled for their responses to expanding stimuli as a function of the mosquitoes' distance to the expanding stimulus

\begin{tabular}{|c|c|c|c|c|}
\hline $\begin{array}{c}\text { Distance to } \\
\text { stimulus } \\
\text { (pixels) }\end{array}$ & $\begin{array}{c}\text { No. } \\
\text { trials }\end{array}$ & $\begin{array}{c}\text { Lower } \\
\text { CI }\end{array}$ & $\begin{array}{c}\text { Upper } \\
\text { CI }\end{array}$ & z-ratio \\
\hline $0-50$ & 1 & 0.141 & 7.1 & 0 \\
\hline $50-100$ & 23 & 15.284 & 34.6 & 15.037 \\
\hline $100-150$ & 45 & 33.599 & 60.3 & 25.536 \\
\hline $151-200$ & 43 & 31.891 & 58 & 24.664 \\
\hline $200-250$ & 56 & 43.096 & 72.8 & 30.123 \\
\hline $250-300$ & 60 & 46.587 & 77.3 & 31.715 \\
\hline $300-350$ & 54 & 41.358 & 70.5 & 29.313 \\
\hline
\end{tabular}


bioRxiv preprint doi: https://doi.org/10.1101/2022.01.08.475512; this version posted January 10, 2022. The copyright holder for this preprint (which was not certified by peer review) is the author/funder. All rights reserved. No reuse allowed without permission.

\begin{tabular}{|c|c|c|c|c|}
\hline $350-400$ & 56 & 43.096 & 72.8 & 30.123 \\
\hline $400-450$ & 75 & 59.81 & 94 & 37.391 \\
\hline $450-500$ & 79 & 63.366 & 98.5 & 38.837 \\
\hline $500-550$ & 38 & 27.65 & 52.2 & 22.424 \\
\hline $550-600$ & 17 & 10.568 & 27.3 & 11.682 \\
\hline $600-650$ & 4 & 1.501 & 10.7 & 2.773 \\
\hline
\end{tabular}

